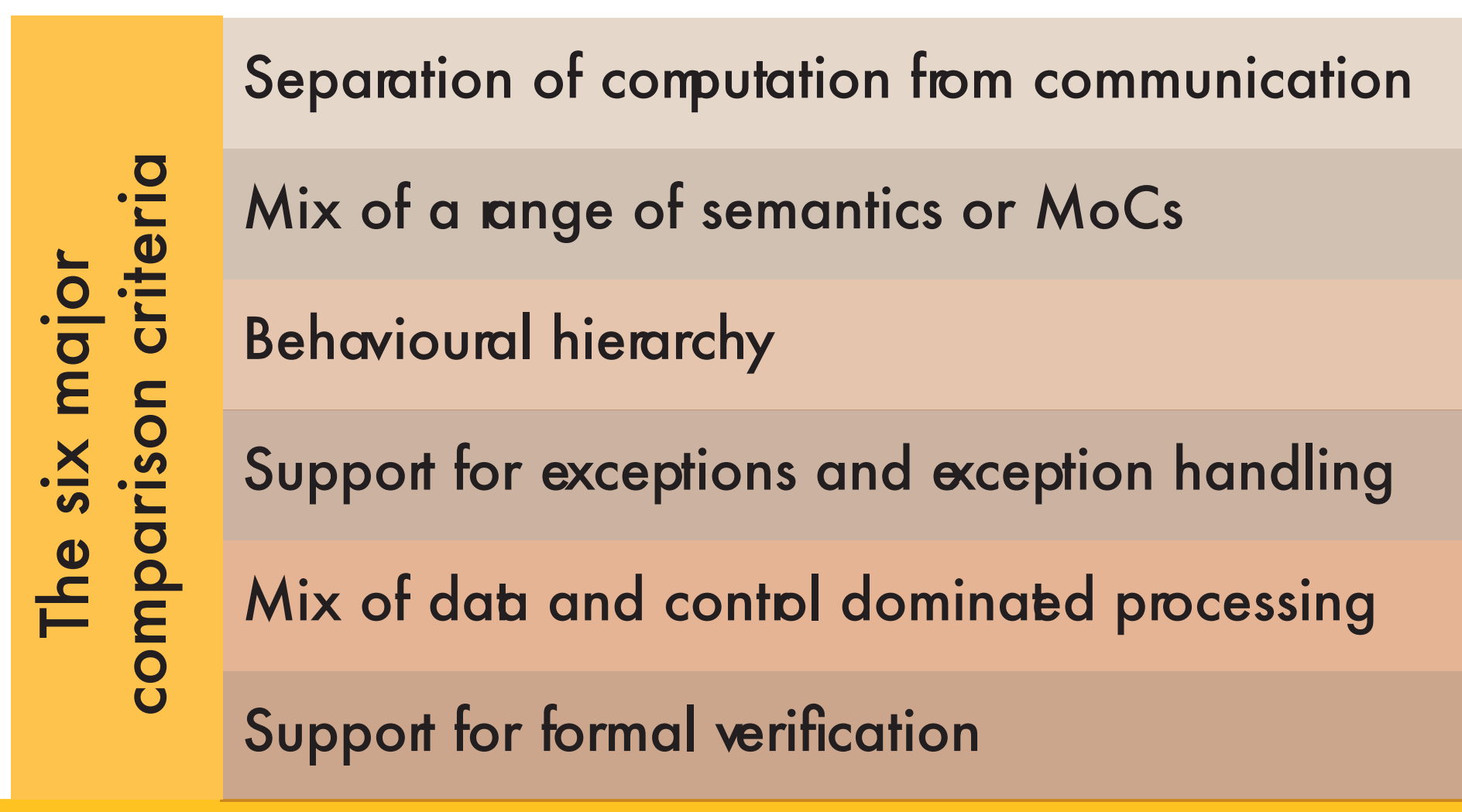

\title{
The SystemJ Approach to System-level Design
}

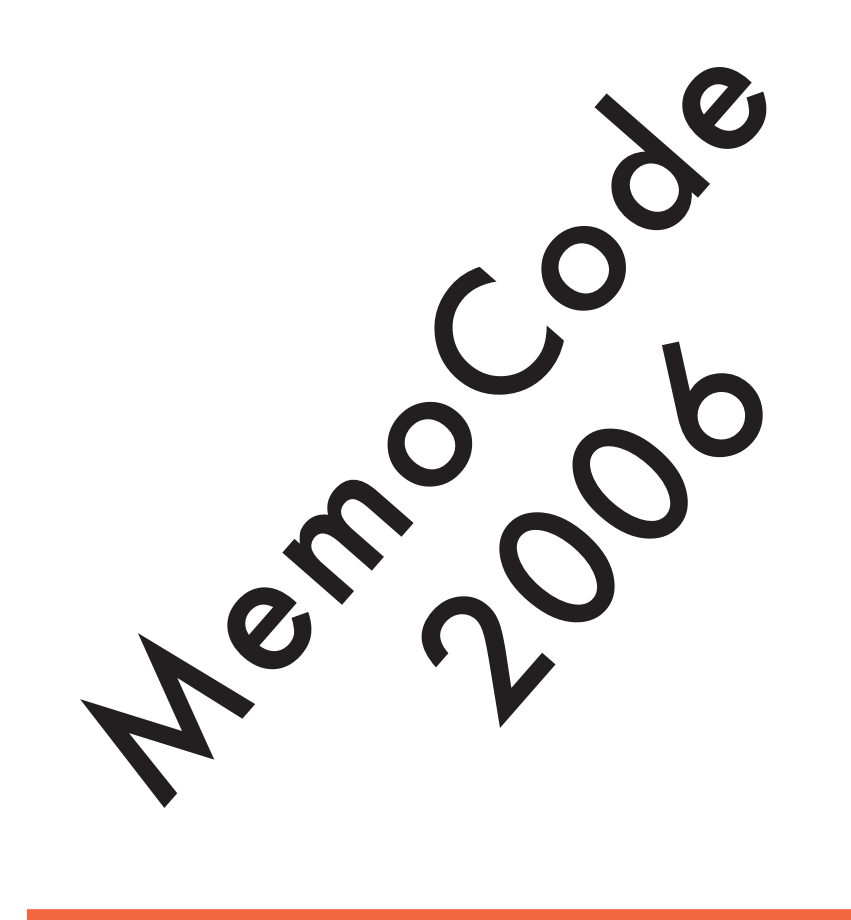

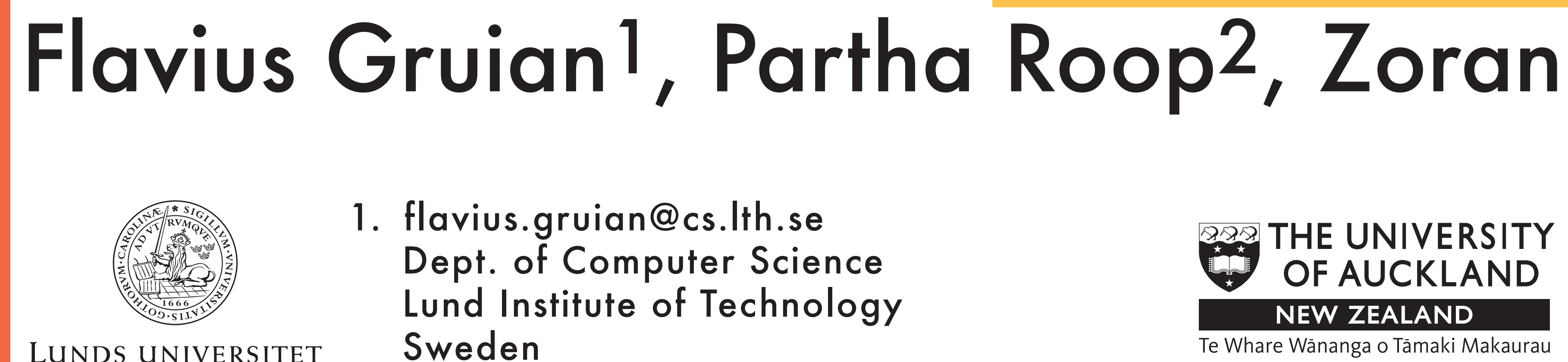
2. \{p.roop,z.salcic,i.radojevic\}@auckland.ac.nz Dept. of Electrical and Computer Engineering The University of Auckland New Zealand

SystemJ, the language

designed for specification, modeling and synthesis of GALS systems

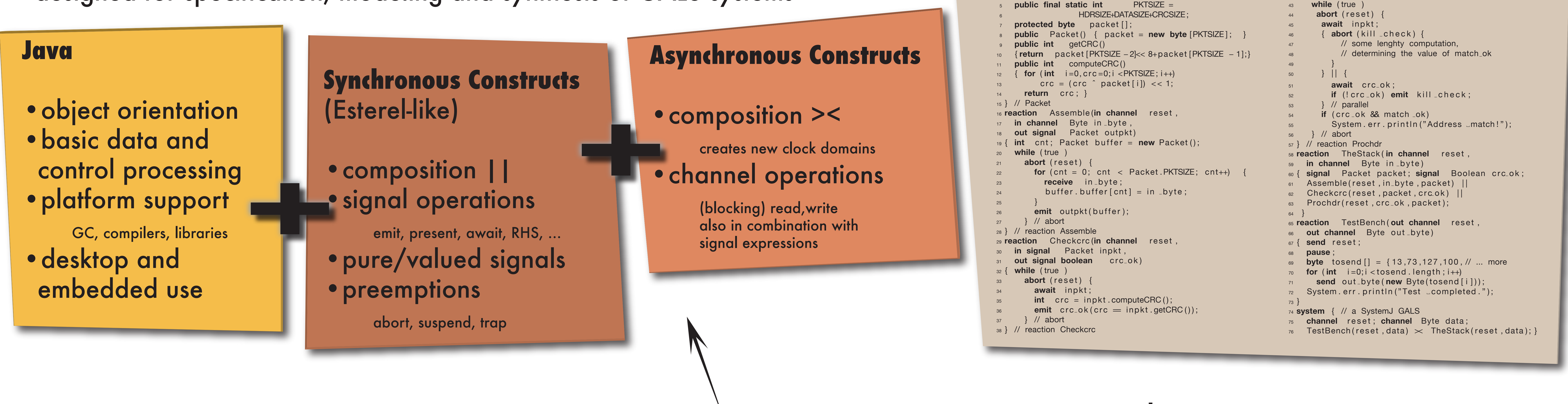

TReK: A True Reactive Kernel Java 1.5 Library

- offers support for desktop execution of SystemJ specifications

- sixteen classes implementing reactions, signals, channels, clock domains, and scheduling

- extends standard Java threads and employs two queves to achieve the synch-asynch behaviour at run-time

- uses try-catch to handle aborts and traps

- signals and signal operations implemented using bit arrays

- employs generics to implement valued signals and channels

cions

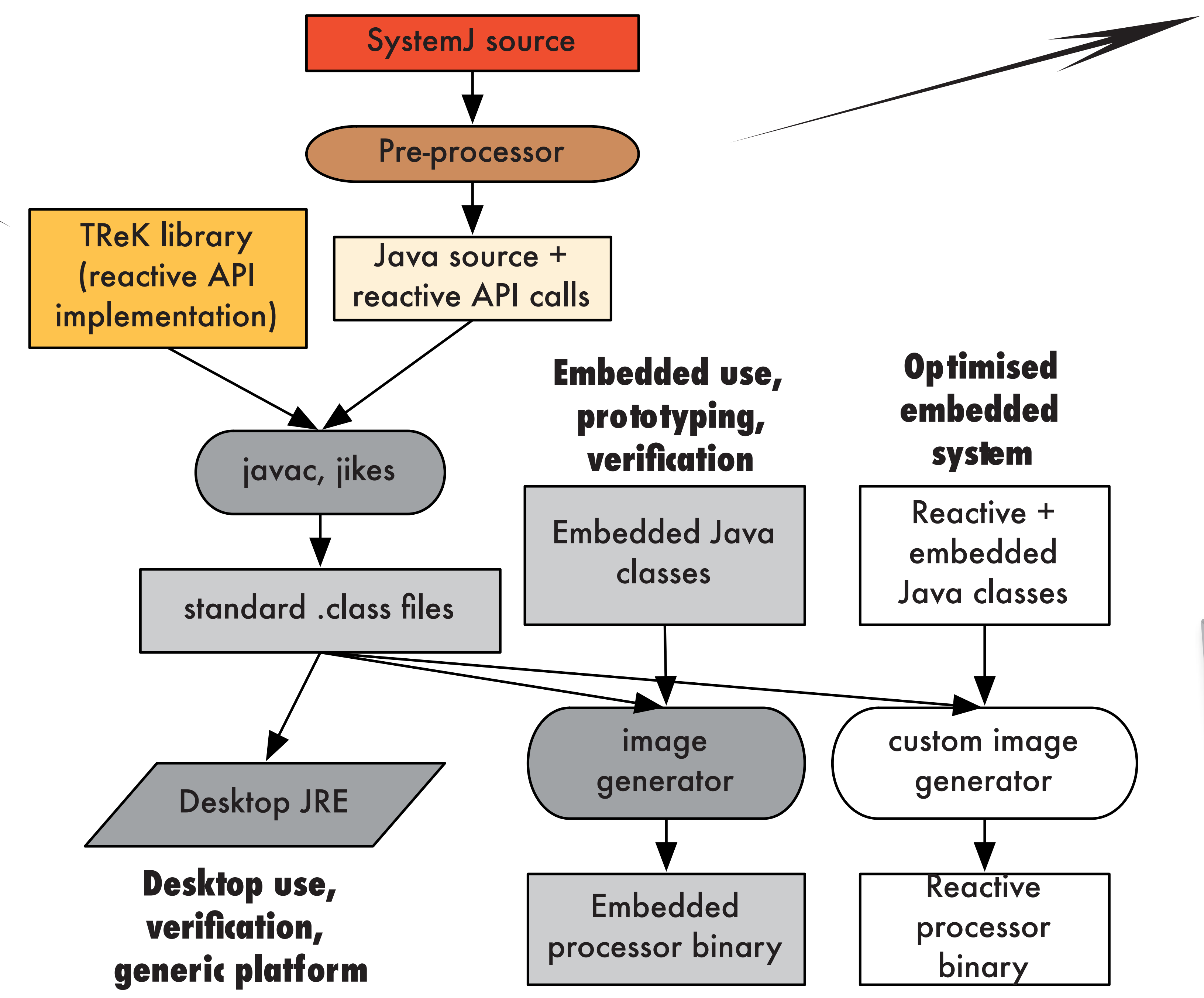

The SystemJ Pre-processor

- translates SystemJ to Java plus TReK calls

- detects syntactic errors, incorrect uses of channels and signals

- introduces signal resolves to help the run-time scheduler

- based on ReRAGs with JastAdd, a Java 1.4 frontend and generates Java 1.5 code

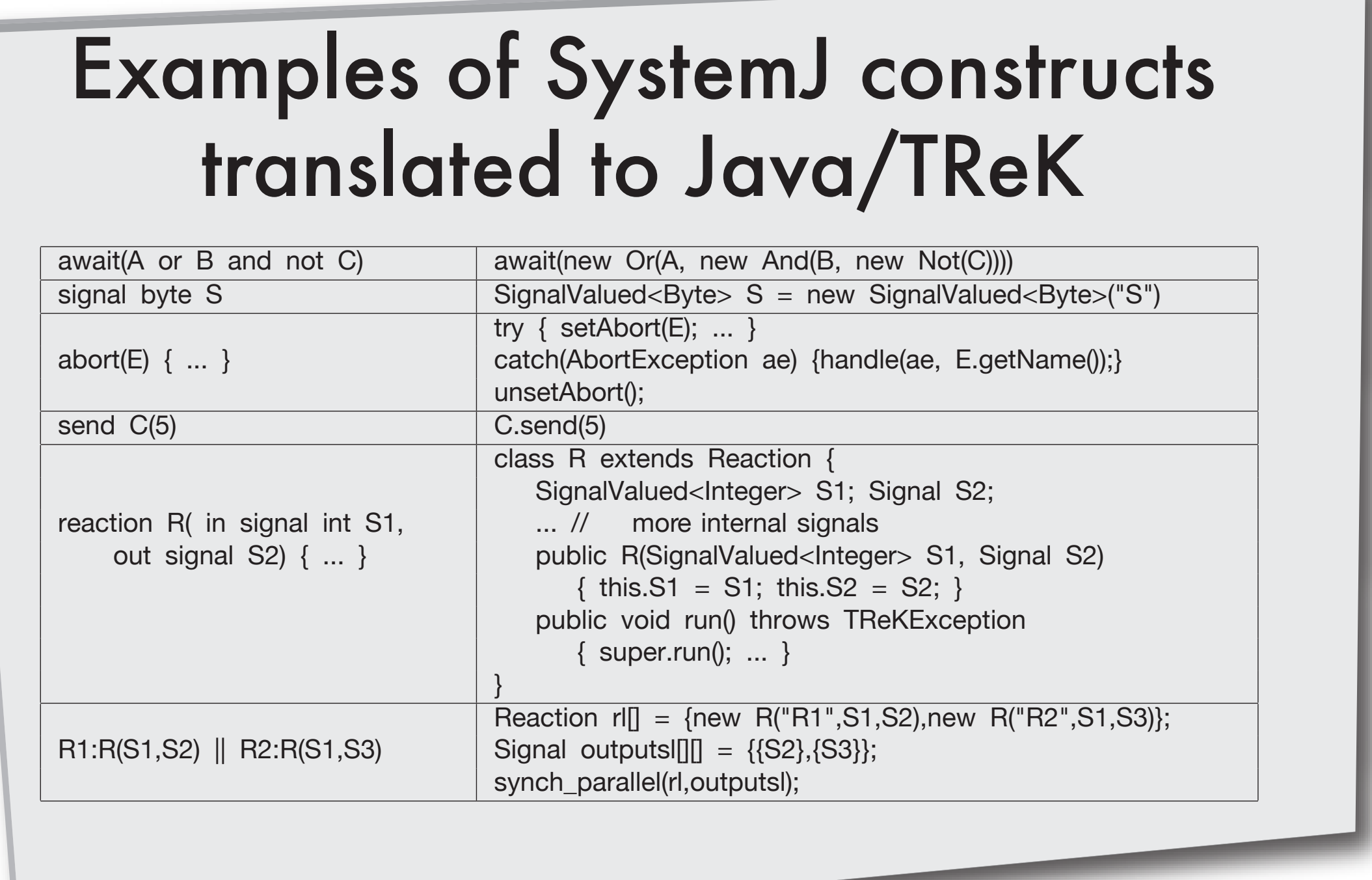

Possible uses of SystemJ (Java based design flows)

\section{Ongoing \& Planned Work}

- reactive Java optimized processor (a JOP-ReMIC mix)

- multi-processor architectures (based on HiDRA and Emperor)

- custom JVM for efficient scheduling

- debug and visualisation tools in Eclipse

- formal semantics

\section{Formal Verification}

- no formal semantics YET

- can be translated to extended CRSM
The equivalent ECRSM of the Protocol Stack Example

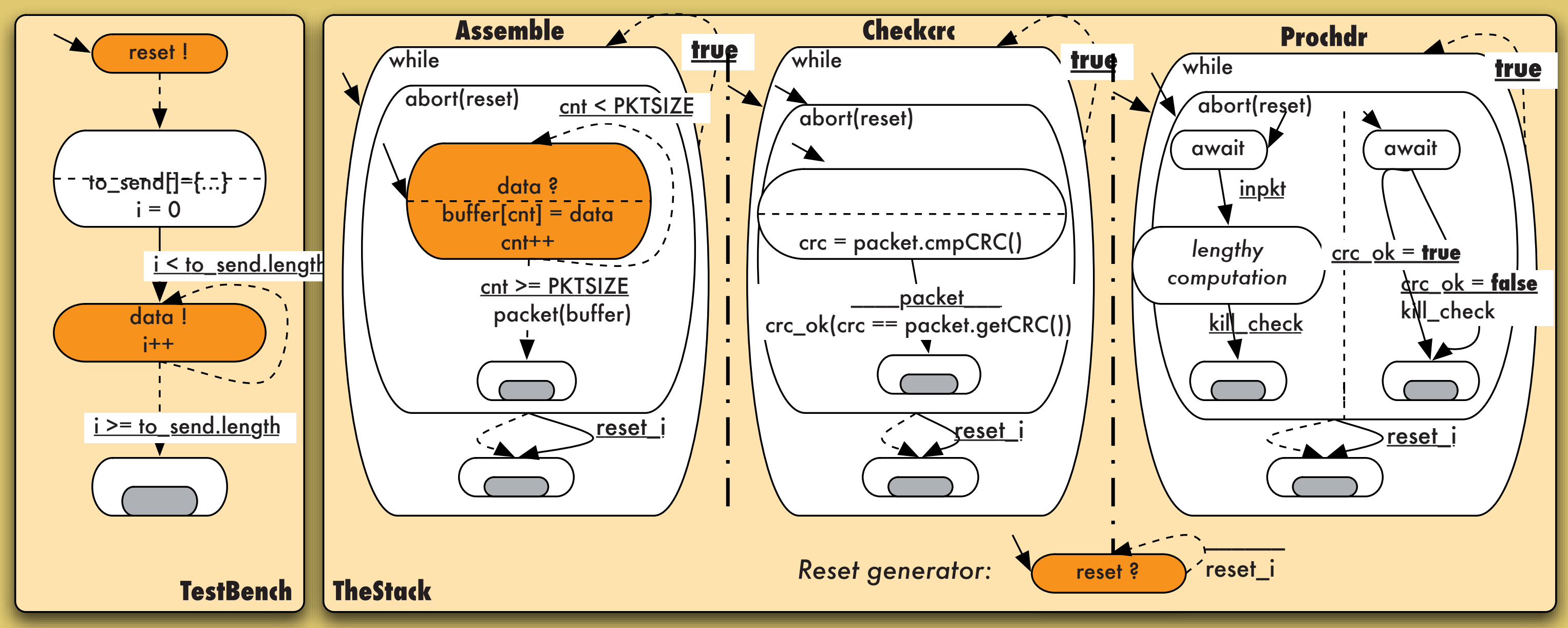

